\title{
DISCUSSION FOLLOWING PAPER BY PACZYŃSKI
}

Iben to Paczyński: We may not be worried if stars in the range $2 M_{\odot}$ to $8 M_{\odot}$ do not make pulsars but, if these stars are detonated and leave no remnant do we not make far too much iron peak matter?

Ostriker to Paczyński: I have some comments on the insights offered by statistical considerations concerning the likely endpoint of stars in the 3-8 $M_{\odot}$ range.

(1) I doubt if enough stars die in the mass range $8<M / M_{\odot}<\infty$ to account for the number of pulsars.

(2) If all stellar cores from stars $3<M / M_{\odot}<8$ were explosively turned into iron peak elements, I think that far more iron would have been produced in the history of the Galaxy than is currently observed.

(3) These statistical arguments have an uncertainty greater than \pm 0.3 to 0.5 in the logarithm so that (1) and (2) do not constrain the theory overly.

Paczyński: I believe that it is better to look at the iron problem in a different way. One should compare the amount of iron ejected in carbon detonation supernovae (if carbon detonates) with the amount of $\mathrm{C}, \mathrm{N}, \mathrm{O}$ produced in massive supernovae discussed by Arnett. In this way we could put an upper limit to the mass range of stars which may be allowed to detonate carbon without violating the observed $\mathrm{Fe} /(\mathrm{C}+\mathrm{N}+\mathrm{O})$ ratio, but I do not believe that we could exclude on observational grounds the possibility of carbon detonation. I am afraid that at present this is purely a theoretical problem and the theoretical answer is uncertain as I tried to emphasise in my review. Indeed, when mass loss is taken into account, it is even possible that the lower mass limit for carbon detonation could be higher than the higher mass limit!

Sugimoto to Paczyński: I would like to give two comments concerning the cause of mass loss. (1) I computed the instability of the helium-burning shell for the stage of C-O core mass very close to the Chandrasekhar limit $\left(1.39 M_{\odot}\right)$. The peak of the energy generation by the helium burning is only $L_{\mathrm{He}} \simeq 10^{7} L_{\odot}$, which is too small to produce any dynamical effect. The reason why the peak is so low is that radiation pressure is so much larger than gas pressure. If we then keep the pressure constant at the helium-burning shell, while the entropy increases, the temperature rises but the density decreases so much that the nuclear energy generation decreases.

(2) Paczyński and Ziolkowski (1968) estimate the upper limit of mass below which the binding energy of a red giant envelope becomes negative and found 3-4 $M_{\odot}$, for the case of mixing length equal to pressure scale height $(l=H)$. Provided the mixing length satisfies $0.7<l / H<2.0$, the value of the upper limit of mass varies slightly. Another mechanism of mass loss will be necessary, if the maximum mass of stars becoming planetary nebulae is to be appreciably larger than $4 M_{\odot}$. 
Arnett to Paczyński: Great care must be taken in estimating the number of high mass stars which may become supernovae.

(1) The 'Salpeter' mass function is sometimes taken as $\psi \sim m^{4 / 3}$ and sometimes as his tabulated version (which has $\psi \sim m^{5 / 3}$ or so for $m \geqslant 10 m_{\odot}$ ). More realistic values similar to the latter are actually used by myself and Talbot in some of our statistical arguments.

(2) One should use direct counts of OB stars rather than the mass function for this question of pulsar formation since it avoids the question of whose luminosity function to mass function conversion you use.

Schwarzschild to Paczyński: I fully agree with Dr Paczyński that the mass ejection from low mass supergiants, particularly the ejection of a planetary nebula, is most likely caused by an envelope instability, not by the interior shell flash instability. Nevertheless, the structure of the envelope varies during an interior flash cycle and there appears to be a particular phase in the interior cycle at which the envelope instability is particularly strong. At this phase the envelope has a fairly unusual thermal structure. If the main part of the ejection of the planetary nebula occurs at this phase, it appears from recent Princeton computations that the remnant star is left in such a special state that it makes its transition from a red supergiant to a blue nucleus very fast $(\sim 3000 \mathrm{yr})$; that is fast enough to properly illuminate the ejected nebula before the latter hopelessly disappears. 